\title{
The Analogy in Decision-Making and the Implicit Association Bias Effect
}

\author{
Nataliia Reva \\ Taras Shevchenko National \\ University of Kyiv, \\ Volodymyrska 64/13 Street, \\ 01601 Kyiv, Ukraine \\ e-mail: natalie.reva@gmail.com
}

\begin{abstract}
:
The author stands that thinking by analogy is a natural instrument human have because of the mirror neurons in our brain. However, is it that infallible to rely on? How can we be sure that our hidden biases will not harm our reflections? Implicit Association Bias (IAB), for instance, is a powerful intruder that affects our understanding, actions, and decisions on the unconscious level by cherishing the stereotypes based on specific characteristics such as ethnicity, sex, race, and so on. To check if there is a correlation between the IAB effect and the people's capacity to reason logically, the author had created an onlinesurvey. The focus was on analogical reasoning and IAB tests concerning the question of gender equality in science and everyday life and age prejudices. Keywords: analogy, analogical thinking, Implicit Association Bias, IAT.
\end{abstract}

\section{Introduction}

Human makes decisions a thousand times a day using different apparatus to help themselves. Often we consider a new situation for the one they know. It is in our nature. From our childhood, babies try to copy their parent's habits, their facial expressions, and gestures, their manner of talking, their walk and posture - the first thing they get from their moms and dads. Cognitive scientists say that this type of behavior is possible thanks to the mirror neurons in our brain, which are focused on finding similar patterns.

Nevertheless, this copying is unconscious. Small child, as well as apes or parrots, imitate what they see and hear without re-thinking. Growing up, they find the new role models and start to analyze and compare their parents with the new idols choosing the elements they like more. At this point, the child begins reason by analogy consciously. 
The ability to spot existing or emerging patterns is one of the most (if not the most) critical skills in intelligent decision making, though we are mostly unaware that we do it all the time [14]. Human brain works by patterns and associations - if a perception fits roughly into an existing pattern, then it may be taken as definitive. We see a half-hidden person dressed or coiffured as someone we may know and "recognize" this person by his/her type of clothes or hairstyle. We distinguish a fake smile from a genuine smile, predict from person's body language if he/she is telling the truth or read from the facial expression what people are thinking about at this particular moment.

This ability of our brain makes our life easier, but at the same time, it leaves some loops. The not only analogy works in this manner. Not so far, American scientists, Kahneman and Tversky, have discovered the whole series of biases that imperceptibly intrude into our decision-making process. For instance, Implicit Association Bias (IAB) arises from the quick automatic association by noticing patterns between two or more similar things, i.e., creates rapid mental connections between the objects, actions, and ideas that share the same patterns as well as the analogical reasoning. In this article, we are going to take a better look at both of them to find their similarities and differences.

\section{Analogy}

In early 80s Dedre Gentner developed the structure-mapping theory according which an analogy is a mapping of knowledge from one domain (the base or source) into another (the target) such that a system of relations that holds among the base objects also holds among the target objects. Meaning that analogy works by establishing the correspondences between two objects, so the new inferences derive by importing connected information from one object to another. To do so, these objects should have some common patterns [4].

Analogical mapping is often used because of its simplicity and familiarity for our brain. However, sometimes, it requires a good level of creative thinking. There is a small number of analogies that can be taken from our memory. Basically, because our memory is not limitless. Besides, it is in human nature to forget things. Thus, the other analogies suggest that we use some "unexpected" sources, like the creativity of our mind. The last one is claimed to be the origin of novelty, which analogical reasoning is glad to benefit from.

Gentner and Loewenstein insisted that analogical reasoning as a reflection process from particular to particular can be divided into simple stages. They distinguished 4 steps that people "pass" reasoning by analogy: (1) retrieval of potentially useful related case given another (2) mapping between two cases in working memory (finding the correspondence/ likeness) (3) evaluating the analogy and its inferences (using a source analog to form a new conjecture) (4) abstracting the common structure (good analogy is structurally consistent). [3]

Holyoak and Thagard supporting Gentner's idea stressed that good analogical reasoning follows three kinds of constraints: similarity, structure, and purpose. They do not operate like rigid rules but assist the internal coherence of the analogical reasoning. Returning to the Little Aaron, his example adheres to all restrictions. Aaron's mom hit her hand as her son done for hundreds of time (similarity). The boy kissed his mom's hand she had done before (structure). Aaron wanted to make his mom feel better by easing her pain by a kiss (purpose) [6].

As straightforward and widespread analogical thinking is, it also proved to be useful. Gick \& Holyoak conducted an experiment whose results reported some curious data. Only $10 \%$ of people who simply read and tried to solve the problem succeeded. While $30 \%$ of those who were given a story with an analogous solution, yet, with different specific content, before receiving the insight problem, solved it. Three times as many as without the analogy! Seems incredible! [9].

Using analogical reasoning in decision-making process simplifies the last one, proposing to use some already established model instead of creating a new laborious resolution. Thus, the more previous experiences one have, the more connections this person easily can make. Analogies may be applied at 
various levels: in the same case or a case with similar structure; in social interaction with the same individual or with individuals who are considered analogous (e.g., are in similar relations to me, like family or team members), etc. [13].

However, if analogies are so quick and easy-made, moreover based on our elusive memory and previous doubtable experience, how can we be sure that analogical reasoning is infallible to rely on? How can we be sure that our hidden biases will not harm our reflections? Does our level of critical and logical thinking preserve us from errors? Marijke Breuning insists that analogies can fail if and only if they are constructed based on superficial similarity, not deep causal traits [1].

\section{Implicit Association Bias}

Implicit stereotyping is systematically studied through well-established methods based upon principles of cognitive psychology that have been developed in nearly a century's worth of work. The IAB is demonstrated in two paradigms: (1) says that the cognitive salience of a familiar stereotype can implicitly bias social judgment in stereotype-consistent ways (Devine's critical experiment); (2) states that social attitudes - including prejudice and stereotypes - are empirically captured by the degree to which they are linked through speed and efficiency to semantically related concept [11].

IAB has the next characteristics: (1) It belongs to the I System of human "Machinery of Thought" that is represented by the quick automatic mode of decision-making. We can identify it with human intuition and instinct [12]; (2) It is unconscious, so humans are unable to catch its presence at once. Just as Freud suggested that we push our sexual troubles and traumas out of consciousness, yet they continue to follow us and have an influence on us in the form of our dreams, linguistic errors or even some kind of depression. Cognitive implicit biases hide in the dark corners of our mind waiting for the right time to show their effect on us; (3) It works on the rapid mental associations attached to people behavior and attitude; (4) It can contradict human conscious beliefs and positions.

Where can we see the manifestation of the IAB? Literally everywhere! It could happen in any domain: recruitment, healthcare, outcomes in criminal justice, etc. For example, if meeting a person for the first time, you, rather than being neutral, have a preference for (or aversion to) he/she based on such characteristics as race, gender, ethnicity, age, or even appearance; this is the manifestation of the implicit association bias. I think anyone of us had in our experience a person who was more loved by our teacher/boss or whoever else only because he/she had some characteristics this person likes or on the contrary you have some flaws this person hates.

However, you should understand that it does not make you or anyone else a racist, sexist, etc., anytime such a stereotype popped into your mind. It just means that your brain is working properly, noticing patterns, and generalizing! Racism or sexism is a decision made by a sharp mind. The implicit associations are 'rogue' processes, which are not properly seen as part of the agent's character - not indicative of 'who she is.' Merely being influenced by implicit bias does not mean that one has the nature of a racist or sexist person; it takes something other than the operation of implicit racial biases to be properly ascribed the character trait racist [7].

IAB have both positive and negative results. From the bright side, IAB facilitates fast-made judgments and decisions. Although it does it by undermining the true intentions, changes the behavior, and sets people up to overgeneralize. For instance, imagine a police officer that believes in his superior role to protect and serve people. He is deeply committed to these principles. Yet, most of the time, he stops only men of color. If you ask him why he is doing that he will not be able to give you a rational answer. The truth is - he is biased! Unconsciously he associates a black or a brown face to the criminal one (without being aware of it). This police officer suffers from the implicit race bias that could have appeared in his childhood or forced by his everyday environment.

At the same time, we can have some harsh prejudices against a government, for instance, associating it with the word corruption, or bankers associating them with greed, or militaries 
associating them with aggression. Thereby when you meet a man, who claims to be a banker, but dreams of going to politics, you may unconsciously associate this desire with his greed and corruption inclinations. That is how the implicit bias can penetrate the human decision-making process and affect or even modify the decision. The good news is that any bias could be corrected by simple re-thinking! When you get to know better this man, you can change your mind, finding him kind and fair, or confirm your first assumptions.

\section{Analogical Reasoning and Implicit Association Bias Experiment}

Our brain is a powerful machine that knows how to simplify the word for us. Just imagine if we would need to think of everything in a logical, meticulous way comparing all the propositions and desires. It would take a lot of time and mental energy. Therefore, our brain constructs a vast amount of models for quick understanding and processing the information in our memory, so we would not have to deal with it later in the future one more time. The same principle works in so-called "Holistic learning," where you learn things by connecting them to other ideas and creating mental constructs of concepts. [17]

Nevertheless, nothing is perfect! Even our brain. That is why the loops open for the biases in the System I become possible. As it was mentioned before, IAB works in the same manner as the analogical reasoning by gathering commonalities together. That means that IAB like a virus or a tree fungus clings to our brain and unnoticed functions with it. Let us sum up in the table below the main characteristics of the analogical reasoning and IAB.

\begin{tabular}{|l|l|}
\hline Reasoning by analogy & Implicit association bias \\
\hline $\begin{array}{l}\text { The reasoning is typically considered with a } \\
\text { high-level awareness and rationality that } \\
\text { belongs to System II. }\end{array}$ & $\begin{array}{l}\text { Manifest themselves using the loops of the } \\
\text { System I, creating rapid mental connections } \\
\text { between the objects, actions, and ideas. }\end{array}$ \\
\hline $\begin{array}{l}\text { Identifies a common relation between two } \\
\text { situations and generates further inferences } \\
\text { driven by these commonalities. }\end{array}$ & $\begin{array}{l}\text { Arises from the quick automatic association by } \\
\text { noticing patterns between two or more similar } \\
\text { things. }\end{array}$ \\
\hline $\begin{array}{l}\text { Consciously makes generalizations to come to } \\
\text { a specific decision. }\end{array}$ & $\begin{array}{l}\text { Unconsciously makes generalizations to come } \\
\text { to a certain decision. }\end{array}$ \\
\hline Rational good analogies are structuralized. & $\begin{array}{l}\text { Unconscious associations are driven by } \\
\text { indefinable emotional impulses. }\end{array}$ \\
\hline
\end{tabular}

As these two phenomena work similarly, the next questions arise: (1) could the Implicit Association Bias intrude our Analogical reasoning? (2) Could the stereotypes or prejudices take the place of the rationally made analogies in the name of fast thinking? My hypothesis is that IAB not only can but also do so quite often. Therefore, there should be a correlation between them. We can assume that if there will be found a robust and significant correlation $(r=$ more than .05$)$ between the level of Analogical 
reasoning and the IAB a person shows, we may say that human analogical reasoning (sufficiently) suffers from the unconscious impulses.

\section{Method}

To check the hypothesis, I have created an online survey on the Lime Survey platform. The study was performed in Ukrainian language, so here I am giving you the translations. It was composed of three parts: two on the analogical reasoning test and one on the implicit association bias. The analogical test part was run twice (before and after the IAB) to see if the implicit association bias mutually with the pressure of time affects human decisions. The study run in the next sequence: (1) First, the participants have as many time as they need to reflect on the ten questions on analogy. (2) After they do the IAB test (that is limited in time) to rate the level of gender and age stereotypes they unconsciously have. (3) Then they have a new analogical test, and they need to answer these questions as quickly as they can accordingly to the timer (10 seconds per question). Besides, in the last task, five questions out of ten concerned the same topics as the IAB test, i.e., have gender and age implication.

The original idea to take the IAT was rejected, firstly, because of its complexity and long timeconsuming; secondly, because the new studies showed its shiftiness. [2, 15] Thus, I decided to create my test that will not take much time and will be simpler to do, yet, that will be still based on the same principle as the IAT - the association test on millisecond reaction time. For example, to see the gender preference unconscious, I named some professions, like an astronaut, first-grade teacher, nurse or mathematician, to the students and asked them to choose to whom it is more suitable - for a girl named Olia and a boy called Tolia. To check the race prejudice, I gave the students some examples of the presents, like a book, laptop or a bike, and asked to choose which of them are good for a son and which for his grandpa. Subjects had only 30 seconds to make their decision.

Additionally, I decided to check Holyoak's assumption that analogical reasoning is a congenital ability. Holyoak \& Thagard gave an example of little Aaron who at his second year of life was able to derive an analogy from to similar situations, that shows that analogical reasoning does not require any tutoring in logic or critical thinking [8]. Thus, I invite not only people who studied logic, but also those who never had a deal with critical thinking. The total number of the participants is 50 (25 from each side). All of them are students from my alma mater - Taras Shevchenko National University of Kyiv. Half of them - the third grade - had attended a course on classical logic. The first grade did not have any logical classes at that time or before.

After the revision, only half questionnaires turned out to be competently and correctly fullfilled. Seven of the participants were male subjects, while eighteen were female subjects. Only two representatives out of 25 said that they prefer men to women in work, while four gave their preference to women. At the same time, 12 represents indicated that they prefer to work with young people and none favored elders. An interesting fact is that all four who suggested their preference to women showed prejudice against them in the question of career and profession.

\section{Conclusion}

Out of 100 answers on modified IAT test, 28 did not show any biased, while 72 were the biased answers. Overall, 50\% of the representatives showed a gender-bias and 70\% - the age-bias! For instance, all the respondents $(100 \%)$ recognized the primary school teacher as a female profession, while $86 \%$ of participants chose the astronaut as a job for men. At the same manner, the subjects decided that the exact sciences, like mathematics and astronomy, are more suitable for men (72\% and $80 \%$ ) while the Humanities and Art fit the women (89\% and 95\%).

Talking about analogical thinking, all subjects made more mistakes in the second part after they pass the IAB test. If we analyze the two analogical tests, out of 250 answers, only 69 were incorrect in 
the first test compared to the 135 in the second one. Looking at these results, we can previously agree with the hypothesis that in time-limited circumstance the implicit association bias easily intrudes the decision-making process and to save time replace the analogical reasoning simultaneously pushing people to make the wrong choices.

Moreover, in the three of five questions that had gender or age stereotypes, the results showed in average $60 \%$ of biased answers. For instance, to the question "apple tree: apple: father:?" only five people gave the right answer "a child" when all the other chose the wrong variant - "a son." At the same way, to the question "fast: slow: immature:?" only three participants selected the correct answer "mature/developed," while seventeen chose "aged," the rest also gave wrong answers "young" or "green," probably not understanding the analogy of this task.

Statistics were done in the SPSS. It reveals a significant correlation (Pearson's $r=.06$ ) between the level of implicit association bias people have and their use of analogical thinking. As a result, the subject's answers showed that the author's hypothesis was right. Besides, preliminary study or nonstudy of logic did not affect the test results. Thus, we can conclude that Holyoak's theory of analogical thinking as natural human ability may have sense. For future researches, the author plans to check it in correlation with the other social biases and heuristics, like, for example, the anthropomorphism.

\section{References}

1. Breuning, M. The Role of Analogies and Abstract Reasoning in Decision-Making: Evidence from the Debate over Truman's Proposal for Development Assistance, International Studies Quarterly 47 (2), 2003, pp. 229-245.

2. Brunel, F., Tietje, B., Greenwald, A. Is the Implicit Association Test a Valid and Valuable Measure of Implicit Consumer Social Cognition? 2003.

3. Gentner, D., \& Loewenstein, J. Learning: Analogical Reasoning. Encyclopedia of Education, Second Edition, New York: Macmillia, 2003.

4. Gentner, D. \& Maravilla, F. Analogical reasoning, In L. J. Ball, V. A. Thompson (eds.), International Handbook of Thinking \& Reasoning, New York: Psychology Press, 2018, pp. 186-203.

5. Gentner, D. \& Smith, L. Analogical reasoning, In V. S. Ramachandran (ed.), Encyclopedia of Human Behavior (2nd Ed.), Oxford: Elsevier, 2012, pp. 130-136.

6. Gick, M., Holyoak, K. J. Analogical Problem Solving, Cognitive Psychology 12, 1980, pp. 306-355.

7. Holroyd, J., Kelly, D. Implicit Bias, Character, and Control, Personality to Virtue, Oxford University Press, 2014.

8. Holyoak, K. J. Analogy and relational reasoning, In K. J. Holyoak, R. G. Morrison (eds.), The Oxford handbook of thinking and reasoning, New York: Oxford University Press, 2012, pp. 234-259.

9. Holyoak, K. J. \& Thagard, P. The Analogical Mind, American Psychologist 52 (1), 1997, pp. 35-44.

10. Hristova, P. Unconscious Analogical Mapping? New Bulgarian University 2009, pp. 655-660.

11. Jost, J. T., Rudman, L. A., Blair, I. V., et al. The existence of implicit bias is beyond reasonable doubt. Review, Research in Organizational Behavior 29, 2009, pp. 39-69.

12. Kahneman, D. Thinking Fast and Slow, New York: Farrar, Straus, and Giroux, 2011.

13. Kokinov, B. Analogy in decision-making, social interaction, and emergent rationality, Behavioral and Brain Sciences 2003.

14. Miemis, V. Essential Skills for 21st Century Survival: Part I: Pattern Recognition, Retrieved from: https://emergentbydesign.com/2010/04/05/essential-skills-for-21st-century-survival-part-i-patternrecognition/.

15. Nagai, A. The Implicit Association Test: Flawed Science Tricks Americans into Believing They Are Unconscious Racists, Special Report no 196, The Heritage Foundation, 2017.

16. Reva, N. Logic, Reasoning, Decision-Making, Future Human Image 10, 2018, pp. 76-84. 
17. Young, S. A Brief Guide to Learning Faster (and Better), 2011, Retrieved from: https://www.scotthyoung.com/blog/2011/01/11/learn-faster-and-better/.

\section{APPENDIX}

\section{Visual analogy tests}

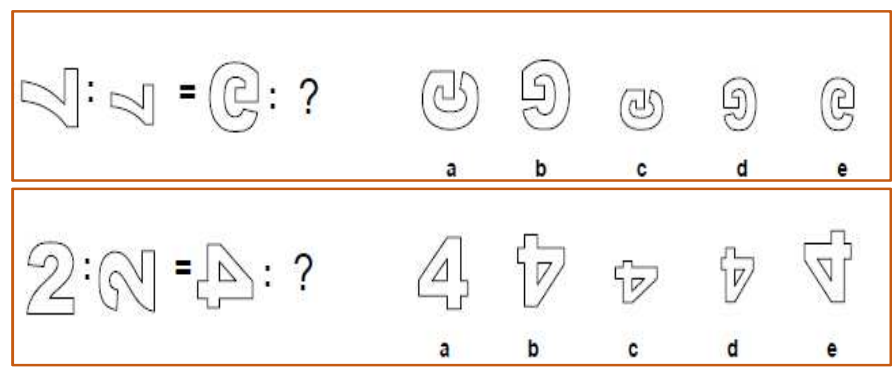

\section{Verbal analogy tests}

\begin{tabular}{|l|l|}
\hline : tradition : hedonist : pleasure & outlaw : ?: offend : affront \\
a. purist (term) & a. chase \\
b. Eden & b. police \\
c. displeasure & c. crime \\
d. Agnostic & d. forbid (synonym) \\
\hline
\end{tabular}

\section{$\underline{\text { IAB analogy test }}$}

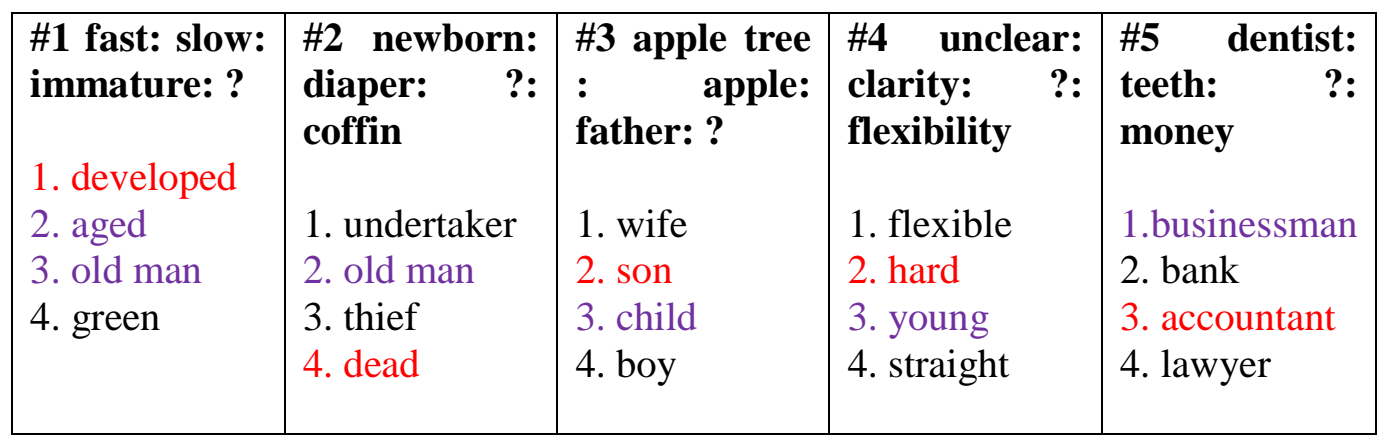

\section{Modified IAT test (30 seconds per question)}

\begin{tabular}{|l|l|}
\hline $\begin{array}{l}\text { Who would you recommend for the } \\
\text { astronaut's position in NASA? }\end{array}$ & $\begin{array}{l}\text { Whom would you recommend to give } \\
\text { a new IPad for a New Year? }\end{array}$ \\
$\begin{array}{l}\text { Olia } \\
\text { Tolia }\end{array}$ & $\begin{array}{l}\text { Grandpa } \\
\text { Son }\end{array}$ \\
\hline
\end{tabular}

